\title{
Tumor FOXP3 Represses the Expression of Long Noncoding RNA
}

\section{SL}

Yanhui Yang ${ }^{1}$, Jingli Cheng ${ }^{1}$, Huizhu Ren ${ }^{1}$, Hui Zhao ${ }^{2}$, Wei Gong ${ }^{3}$, Chunyan Shan ${ }^{1 \#}$

1. 2011 Collaborative Innovation Center of Tianjin for Medical Epigenetics, Key Laboratory of Hormones and Development (Ministry of Health), Metabolic Diseases Hospital\& Tianjin Institute of Endocrinology, Tianjin Medical University, Tianjin 300070,China

2. Tianjin Key Laboratory of Food Biotechnology, School of Biotechnology and Food Science, Tianjin University of Commerce, 300134, China

3. Institute of Radiation Medicine of Chinese Academy of Medical Science and Peking Union Medical College, Tianjin, 300192 , China

\# Corresponding author: Chunyan Shan

2011 Collaborative Innovation Center of Tianjin for Medical Epigenetics, Key Laboratory of Hormones and Development (Ministry of Health), Metabolic Diseases Hospital\& Tianjin Institute of Endocrinology, Tianjin Medical University, Tianjin 300070, China

Tel: +0086-22-23333221

Fax: +0086-22-23333221

Email: chunyanshan15@gmail.com 


\section{Abstract}

The long noncoding RNA 7SL was over-expressed in tumor cells to promote cell growth through repressing translation of P53. However, the regulatory mechanism of 7SL remains to be defined. FOXP3 was identified as a suppressor in several tumors in addition to be a marker of regulatory $\mathrm{T}$ cells. In this study, we detected that over-expression of FOXP3 repressed the transcription of 7SL RNA and contributed to inhibiting tumor growth. Knock down of FOXP3 in MCF-10A normal mammary breast cells up-regulated the transcription of 7SL RNA. Chromatin Immuno-precipitation (ChIP) analysis showed that FOXP3 directly bound to the Forkhead/HNF-3 domain DNA binding sites (-789 to -795$)$ relative to the transcription start site. Meanwhile, Luciferase analysis showed that FOXP3 repressed the full-length 7SL promoter activity, but this suppressive effect was reversed after mutation of the FOXP3 binding site. Further studies showed that FOXP3 promoted the expression of P53 at translational levels through repressing 7SL RNA. In conclusion, this study suggests that 7SL RNA is a direct target of FOXP3 and may be involved in the formation of FOXP3/P53 feedback loop.

\section{Key Words}

Long noncoding RNA; 7SL; FOXP3; P53 


\section{Introduction}

Long noncoding RNAs (lncRNAs above 200 nucleotides) have been identified as multi-functional regulators of gene expression at different levels such as chromatin remodeling and post-transcriptional control[1]. Recent studies support that lncRNAs play key roles in almost all stages of tumor progression and have a potential to be a therapeutic targets and biomarker for diagnosis[2]. The lncRNA 7SL was initially defined as a part of ribonucleoprotein complex (RNP) with six signal-recognition proteins (SRPs). The RNP functions to translocate proteins to the endoplasmic reticulum[3]. 7SL was found to be over-expressed in many tumors[4] and promoted growth of cancer cells by repressing P53 translation[5]. However, the regulatory mechanism of 7SL in pancreatic cancer remains to be defined.

FOXP3, initially identified as a functional marker of regulatory $\mathrm{T}$ cells, was also expressed in tumor cells and identified as a suppressor in several tumors [6]. In breast cancer, FOXP3 inhibited tumor growth through directly trans-activating the expression of tumor suppressor genes [7] and repressing oncogenes [8,9]. Interestingly, recent evidence showed that FOXP3 regulated the transcription of miR-146 to indirectly repress the transcription of NF-kB [10].

Up to now, FOXP3-targeted miRs and long noncoding RNAs are largely unknown. As a transcription factor, Foxp3 binds to the Foxhead/HNF-3 domains of target gene promoters to regulate transcription. Through analyzing the 7SL RNA promoter regions, we found several Foxheas/HNF-3 motifs, triggering us to identity whether FOXP3 directly regulated the expression of 7SL RNA. Since P53 is a downstream 
target of 7SL RNA, we further identify whether FOXP3 promoted the translation of P53 through repressing 7SL RNA, which might be a new mechanism of FOXP3 to regulate tumor growth.

\section{Methods}

\subsection{Cell culture and treatments}

MCF-7 (breast cancer), MCF-10A (mammary epithelial cell line), HeLa (Cervical cancer), HCT-116 (Colon cancer), PANC-1 (pancreatic cancer) was obtained from American Type Culture Collection. MCF-7, HeLa, and HCT-116 cells were cultured in Dulbecco's modified Eagle's medium (DMEM) containing 5\% fetal bovine serum (FBS), HCT116 cells were in McCoy's 5A medium containing 10\% FBS. MCF-10A cells were cultured under DMEM/F12 containing 5\% Horse serum, EGF $(20 \mu \mathrm{g} / \mathrm{ml})$ and Insulin $(10 \mu \mathrm{g} / \mathrm{ml})$.

\subsection{Western blot}

Whole-cell extracts were prepared by lysing cells with SDS lysis buffer supplemented with proteinase inhibitors cocktail (Sigma). Protein lysates (20 mg) were separated by SDS-PAGE, and target proteins were detected by western-blot with antibodies of FOXP3 (ebioscience), $\beta$-actin (Sigma) and P53 (Santa cruz), in conjunction with chemiluminescence detection kits (Amersharn Biosciences).

\subsection{Plasmids construction and transfection}


7SL RNA over-expression plasmids were constructed as previously described [5]. pGEM-Foxp3 over-expression plasmids were obtained from Sino Biological Inc. To achieve knock-down of FOXP3, we chose two effective shRNA targeting FOXP3 (shFOXP3-1, 993-1013nucleotidesfrom transcription starting site and shFOXP3-2,1355-1375)[7] cloned into pSIREN-RetroQ vectors (Clontech) to generate siRNA following the manufacturer's protocol. Plasmids were transfected into the cells with DreamFect reagent $(\mathrm{Oz}$ Biosciences) following the manufacture's protocol.

\subsection{Real time PCR}

Real time PCR analysis was performed as described [5]. Briefly, total RNA was extracted from cells using Trizol (Invitrogen) and cDNA was synthesized using random hexamers and Maxima reverse transcriptase (Fermentas). Real time quantitative analysis was performed by SYBR Green PCR Master Mix (Qiagen) and specific primers according to published sequences: Foxp3 and P21[7], 7SL RNA and P53 [5], SKP2[11].

\subsection{Chromatin Immunoprecipitation analysis}

Chromatin Immunoprecipitation (ChIP) analysis was performed by a commercial kit (Upstate Biotechnology) following the manufacture's protocol. Briefly, cells transfected with FOXP3 plasmids were sonicated and fixed with $1 \%$ paraformaldehyde. Anti-FOXP3 antibody or anti-IgG (Santa Cruz) were performed to 
pull down chromatin. The precipitated DNA fragments were quantified by real time PCR after normalization to the genomic DNA products. The PCR primers were as follows: ChIP1 Left: ACG GCA CCT AAA ACT GGCT A; Right: TCT GAG CAT TCG ACC AAG AG. ChIP2 Left: GAG TGC GAC TCC GAC TCA A; Right: CGA ACT CCT CCT GGT CTC AG. ChIP3 Left: CAC GCC TGT AAT CTC AGC AC; Right: TGC CTC AGC CTC CTG AAT AG. ChIP4 Left: TTC AGA AGC CAA AAG TTT CA; Right: CCA AAA TGT TTG AAA GCG TC. CHIP5 Left: CAG AGG GAG ACC CAA TTT C; Right: CTG TGA AAC TTT TGG CTT CTG.

\subsection{Luciferase analysis}

The promoter region of the human 7SL RNA gene, spanning from +1 to -2100 relative to the transcription start site was amplified by polymerase chain reaction (PCR) and inserted into pGL3-Basic vectors (pGL3-7SL). All constructs were identified by sequencing. Luciferase activity was measured using the Dual-Luciferase Reporter Assay System (Promega) as described previously [12]. The Phusion Site-Directed Mutagenesis Kit (Thermo Fisher) was used for mutation of FOXP3 binding sites.

\section{Statistical analysis}

Mean values were compared by Student's t test or ANOVA. All values were two sided. Analyses were performed using the SPSS17.0 statistical analysis software. Each experiment was done in triplicate and values are presented as mean $\pm \mathrm{SD}$. P>0.05 was considered significant. 


\section{Results and Discussion}

\subsection{Over-expression of FOXP3 decreased the expression of 7SL RNA}

We initially detected whether over-expression of FOXP3 affected the expression of 7SL RNA. Human MCF-7 breast cancer cells have weak expression of FOXP3. After we transfected pGEM-FOXP3 over-expression plasmids into the cells, western blot showed an increased expression of FOXP3 compared with the wide type and the vector control group (Fig.1A). Furthermore, real-time PCR showed that 7SL RNA levels were decreased with the over-expression of FOXP3 (Fig.1B), suggesting that FOXP3 repressed the transcription of 7SL RNA. Furthermore, we also observed that over-expression of FOXP3 decreased the expression of 7SL RNA in a series of cancer cell lines from cervix, pancreatic and colon cancer (Fig. S1), indicating that this repressive effect is not cell-type specific. It is well established that FOXP3 inhibited the growth of MCF-7 breast cancer cells, but the underlining mechanism remains to be defined. 7SL was highly expressed in several cancers including breast cancer [4] to promote cell growth. To further identify whether 7SL is involved in the repressive effect of FOXP3 on breast cancer, we co-transfected FOXP3 and 7SL into MCF-7 cells. As shown in Fig.1C\&D, over-expression of FOXP3 decreased MCF-7 cell growth at different time point, but co-transfection of 7SL counteracted this inhibitory effect, suggesting that FOXP3 inhibited the growth of breast cancer cells, at least in part, through repressing 7SL RNA. 


\subsection{Knockdown of FOXP3 promoted the transcription of 7SL RNA}

Previous studies have shown that normal mammary epithelial cells have higher levels of FOXP3 and lower levels of 7SL RNA compared to the malignant cells [5] [8]. To determine whether FOXP3 inhibited the transcription of 7SL RNA at normal cells, we knocked down the expression of FOXP3 in MCF-10A immortalized human mammary epithelial cells with short hairpin RNA (shRNA) sequences as reported previously [7]. To avoid the off-target effect, we introduced two shRNAs (shFOXP3-1 and shFOXP-2), both of which showed effective inhibition of FOXP3 protein expression in MCF-10A cells by western blot analysis (Fig. 2A). Real-time PCR analysis showed that with the knockdown of FOXP3 (Fig. 2B), 7SL RNA transcription was significantly increased (Fig. 2C). As a positive control, SKP2 gene transcription was also increased (Fig. 2D), in consistence with a previous report [8].

4.3 FOXP3 bound to the promoter of 7SL RNA and directly repressed the transcription of 7SL RNA

In this study, both gain-of-function and loss-of-function studies showed that FOXP3 repressed the expression of 7SL RNA. To the best of our knowledge, this is the first long noncoding RNA identified as a downstream target of FOXP3. In addition to directly controlling gene transcription, FOXP3 has different methods to regulate gene expression. FOXP3 could interact with multiple transcription factors relating to cell growth, differentiation and immune response [13]. FOXP3 may also function as a transcription co-repressor to inhibit the transcription of NF-kB, CREB and RORa 
independent of FKH DNA binding domain [14]. Thus, we should further identify whether 7SL RNA is a direct target of FOXP3. Through screening the promoter regions (from -1 to -2100 ) of 7SL RNA, we found five potential bindings sites of FOXP3 (Fig. 3A). Chromatin Immunoprecipitation (ChIP) analysis was performed to identify the interaction of FOXP3 with the 7SL RNA promoter. MCF-7 cells transfected with FOXP3 plasmids were fixed with paraformaldehyde and sonicated to DNA fragments. After precipitated by FOXP3 antibodies or control IgG, the genomic DNA fragments were subject to quantitative Real time PCR using the primers covering different FOXP3 binding sites (Fig. 3A). The precipitated DNA was quantified relative to the percentage of input DNA, after subtraction to the small amount of DNA precipitated by IgG. As shown in Fig. 3B, the primers covering the first FOXP3 binding sites (FBS1, -789 to -795 ) produced the most amounts of DNA fragments ( $6 \%$ of input DNA), and the primers covering the second FBS (FBS2, -1396 to -1402$)$ produced a moderate level of DNA fragments (2\% of input DNA). In contrast, those primers covering other FBS domains did not produce any signal. To further determine whether the binding of FOXP3 to the 7SL RNA promoter could suppress the transcription, we transfected the full length 7SL RNA promoter luciferase constructs into MCF-7 cells with or without over-expression of FOXP3 and detected the firefly luciferase activity after normalization to the renilla luciferase activity. As shown in Figure 3C, ectopic expression of FOXP3 suppressed the expression of 7SL RNA, even after mutation of the FBS2. In contrast, mutation of FBS1 significantly reversed the repressive effect of FOXP3 on 7SL RNA expression. 
In conclusion, FOXP3 binds to the FBS located at -789 to -795 relative to the transcription start site (TSS), and directly suppresses the transcription of 7SL RNA.

\subsection{FOXP3 induced translation of P53 through 7SL RNA}

The IncRNA-mRNA interactions are important mechanisms to affect mRNA fate. Although the lncRNA 7SL has been found over-expressed in cancer cells for many years, the function of 7SL remains unclear until recently defined as a suppressor of P53 to promote tumor growth [5]. Further studies showed that the suppressive effect of 7SL RNA was independent of the SRP function but through competitively inhibiting the binding of HuR to P53. Since we have identified 7SL as a direct downstream target of FOXP3, it is reasonable that FOXP3 may promote the translation of P53 through repressing 7SL RNA. To identify the regulatory mechanism of P53, we first performed real time PCR and found that over-expression of FOXP3 in MCF-7 cells did not affect the transcription of P53 at mRNA levels. As a positive control, P21 mRNA was significantly up-regulated following the ectopic expression of FOXP3 in MCF-7 cells (Fig. 4A). In contrast, over-expression of FOXP3 significantly increased the protein level of P53, suggesting that post-transcriptional mechanism exists in the regulation of P53. Interestingly, when 7SL RNA was over-expressed in MCF-7 cells, FOXP3-induced translation of P53 was significantly weakened, suggesting that 7SL is involved in the regulatory mechanism of P53 by FOXP3 (Fig. 4B). P53 is a well-defined tumor suppressor to be involved in cell cycle arrest, apoptosis and senescence [15][16]. Interestingly, a recent study demonstrated 
that P53 directly activated the transcription of FOXP3 in response to DNA damage stimuli in breast cancer [17]. We postulate that a positive feedback loop exists between P53 and FOXP3 to inhibit tumor growth, at least in breast cancer.

In summary, we demonstrate that FOXP3 directly represses the transcription of the lncRNA 7SL through binding to the FBS (-789 to -795) located at the promoter region of 7SL, and subsequently enhances the translation of P53. Reconstruction of P53/FOXP3 feedback loop could inhibit tumor growth and represent promising cancer treatment strategies.

\section{Acknowledgment}

This work was supported in part by grants from National Science Foundation of China (No. 81473472 and No. 81172837). 


\section{Reference:}

[1] J.H. Yoon, K. Abdelmohsen, M. Gorospe, Posttranscriptional gene regulation by long noncoding RNA, J Mol Biol 425 (2013) 3723-3730.

[2] S.W. Cheetham, F. Gruhl, J.S. Mattick, M.E. Dinger, Long noncoding RNAs and the genetics of cancer, Br J Cancer 108 (2013) 2419-2425.

[3] D. Labuda, E. Zietkiewicz, Evolution of secondary structure in the family of 7SL-like RNAs, J Mol Evol 39 (1994) 506-518.

[4] R.J. White, RNA polymerase III transcription and cancer, Oncogene 23 (2004) 3208-3216.

[5] K. Abdelmohsen, A.C. Panda, M.J. Kang, R. Guo, J. Kim, I. Grammatikakis, J.H. Yoon, D.B. Dudekula, J.H. Noh, X. Yang, J.L. Martindale, M. Gorospe, 7SL RNA represses p53 translation by competing with HuR, Nucleic Acids Res 42 (2014) 10099-10111.

[6] T. Triulzi, E. Tagliabue, A. Balsari, P. Casalini, FOXP3 expression in tumor cells and implications for cancer progression, J Cell Physiol 228 (2013) 30-35.

[7] R. Liu, L. Wang, G. Chen, H. Katoh, C. Chen, Y. Liu, P. Zheng, FOXP3 up-regulates p21 expression by site-specific inhibition of histone deacetylase 2/histone deacetylase 4 association to the locus, Cancer Res 69 (2009) 2252-2259.

[8] T. Zuo, R. Liu, H. Zhang, X. Chang, Y. Liu, L. Wang, P. Zheng, Y. Liu, FOXP3 is a novel transcriptional repressor for the breast cancer oncogene SKP2, J Clin Invest 117 (2007) 3765-3773.

[9] T. Zuo, L. Wang, C. Morrison, X. Chang, H. Zhang, W. Li, Y. Liu, Y. Wang, X. Liu, M.W. Chan, J.Q. Liu, R. Love, C.G. Liu, V. Godfrey, R. Shen, T.H. Huang, T. Yang, B.K. Park, C.Y. Wang, P. Zheng, Y. Liu, FOXP3 is an X-linked breast cancer suppressor gene and an important repressor of the HER-2/ErbB2 oncogene, Cell 129 (2007) 1275-1286.

[10] R. Liu, C. Liu, D. Chen, W.H. Yang, X. Liu, C.G. Liu, C.M. Dugas, F. Tang, P. Zheng, Y. Liu, L. Wang, FOXP3 Controls an miR-146/NF-kappaB Negative Feedback Loop That Inhibits Apoptosis in Breast Cancer Cells, Cancer Res 75 (2015) 1703-1713.

[11] S. Radke, A. Pirkmaier, D. Germain, Differential expression of the F-box proteins Skp2 and Skp2B in breast cancer, Oncogene 24 (2005) 3448-3458.

[12] H. Ren, T. Zhao, X. Wang, C. Gao, J. Wang, M. Yu, J. Hao, Leptin upregulates telomerase activity and transcription of human telomerase reverse transcriptase in MCF-7 breast cancer cells, Biochem Biophys Res Commun 394 (2010) 59-63.

[13] T.J. Sadlon, B.G. Wilkinson, S. Pederson, C.Y. Brown, S. Bresatz, T. Gargett, E.L. Melville, K. Peng, R.J. D'Andrea, G.G. Glonek, G.J. Goodall, H. Zola, M.F. Shannon, S.C. Barry, Genome-wide identification of human FOXP3 target genes in natural regulatory T cells, J Immunol 185 (2010) 1071-1081.

[14] J. Du, C. Huang, B. Zhou, S.F. Ziegler, Isoform-specific inhibition of ROR alpha-mediated transcriptional activation by human FOXP3, J Immunol 180 (2008) 4785-4792.

[15] K.H. Vousden, X. Lu, Live or let die: the cell's response to p53, Nat Rev Cancer 2 (2002) 594-604.

[16] S.L. Harris, A.J. Levine, The p53 pathway: positive and negative feedback loops, Oncogene 24 (2005) 2899-2908.

[17] D.J. Jung, D.H. Jin, S.W. Hong, J.E. Kim, J.S. Shin, D. Kim, B.J. Cho, Y.I. Hwang, J.S. Kang, W.J. Lee, Foxp3 expression in p53-dependent DNA damage responses, J Biol Chem 285 (2010) 7995-8002. 


\section{Figure legends}

\section{Fig. 1. Over-expression of FOXP3 inhibits 7SL RNA Transcription.}

MCF-7 cells (Wide Type, WT) were transfected with pGEM-T vector or pGEM-FOXP3 plasmids and performed (A) Western Blot analysis. $\beta$-actin was used as an internal control. (B) Real time RT-PCR. Results were expressed as an inhibitory rate relative to the WT group after normalization to the $18 \mathrm{~S}$ rRNA levels. (C) MCF-7 cells were co-transfected with pGEM-FOXP3 and pcDNA-7SL plasmids for 48 hours and cell numbers were quantified by Countless ${ }^{\mathrm{TM}}$ automatic cell counter. Results were expressed as an inhibitory rate relative to the vector control group. (D) Growth genetics of MCF-7 cells transfected with different plasmids for 4 days. Results ( $n=3$ ) were expressed as mean (column) $\pm \mathrm{SD}$ (bar)

\section{Fig. 2. Knockdown of FOXP3 enhanced the transcription of 7SL RNA.}

MCF10A cells were transfected with scramble or shRNA targeting FOXP3 for 10 days. (A) The protein levels of FOXP3 was determined by western blot by specific antibodies. $\beta$-actin was used as an internal control. (B) The mRNA level of FOXP3 was determined by Real time RT-PCR. Results were expressed as an inhibitory rate relative to the scramble control group after normalization to the $18 \mathrm{~S}$ rRNA levels. (C) \&(D) The mRNA level of 7SL and SKP2 was determined by Real time RT-PCR. Results were expressed as a fold induction relative to the scramble control. Results $(\mathrm{n}=3)$ were expressed as mean (column) \pm SD (bar). 
Fig. 3. FOXP3 directly repressed the transcription of 7SL RNA.

(A) Diagram of the 7SL promoter region including five FOXP3 binding sites (FBS)

(B) Chromotin immunoprecipitation (ChIP) analysis. The DNA products precipitated by anti-FOXP3 antibodies were amplified by Real time PCR. Results were expressed as the percentage relative of the DNA products form input genomic DNA, after stustraction to those precipitated by IgG antibodies. (C) Luciferase analysis. Either vector or FOXP3 plasmids were transfected into MCF-7 cells with wide type (WT) or mutated 7SL promoter luciferase reporters. The renilla plasmids were transfected as an internal control. After transfection for 48 hours, cells were lysed and performed dual luciferase analysis. Results $(n=3)$ were expressed as the inhibitory rate relative to vector group and expressed as mean (column) $\pm \mathrm{SD}$ (bar).

\section{Fig. 4. FOXP3 promoted P53 translation through repressing 7SL.}

(A) Real time RT-PCR. MCF-7 cells were transfected with pGEM vector or pGEM-FOXP3 plasmids. After transfection for 48 hours, mRNA levels of P53 and P21 (positive control) were quantified by real time RT-PCR. Results were expressed as fold induction relative to the vector group, after normalization to the $\beta$-actin internal control. (B) Western blot. pCDNA or pCDNA-7SL plasmids were transfected into MCF-7 cells with pGEM or pGEM-FOXP3 plasmids, and the protein expression were blotted by anti-FOXP3, anti-P53 or anti- $\beta$-actin antibodies. 
A
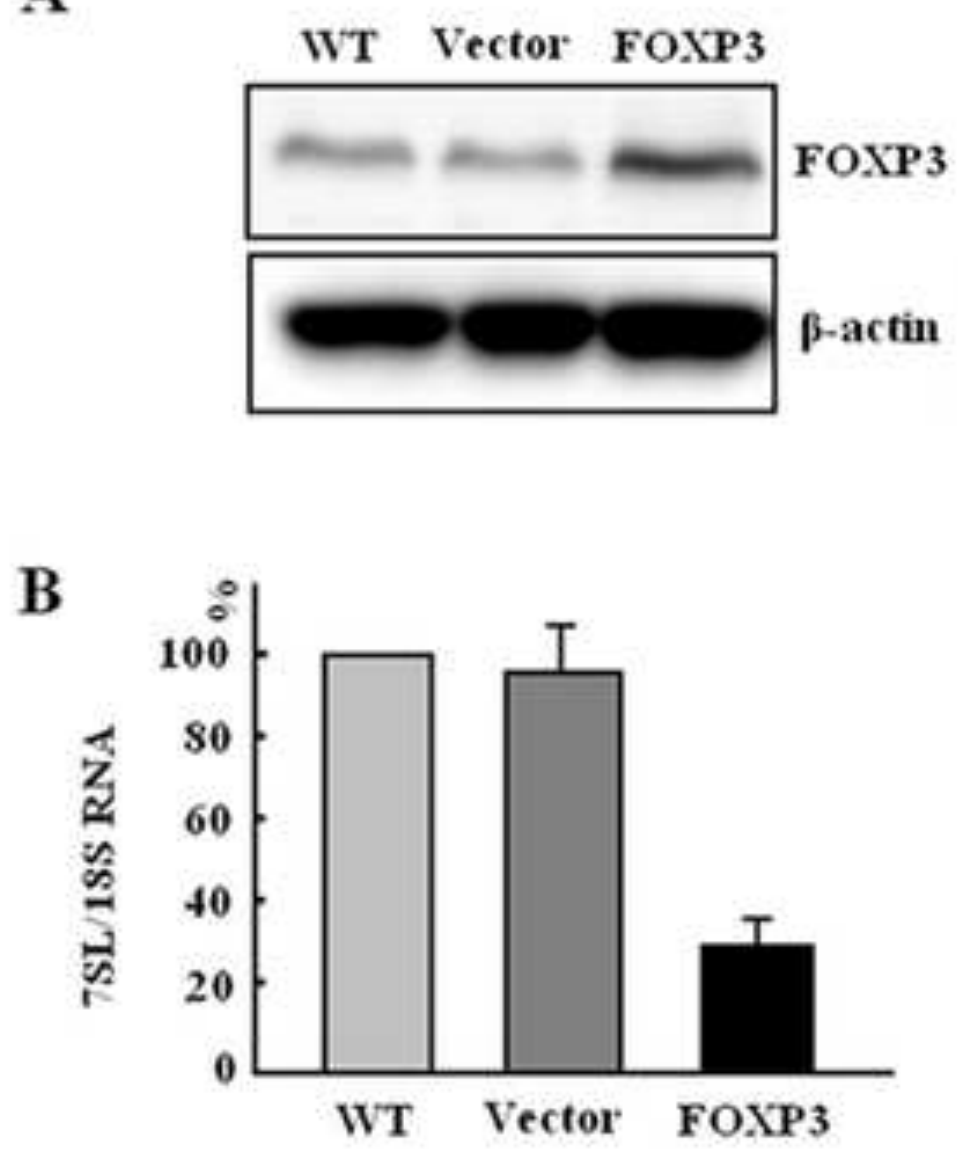

C

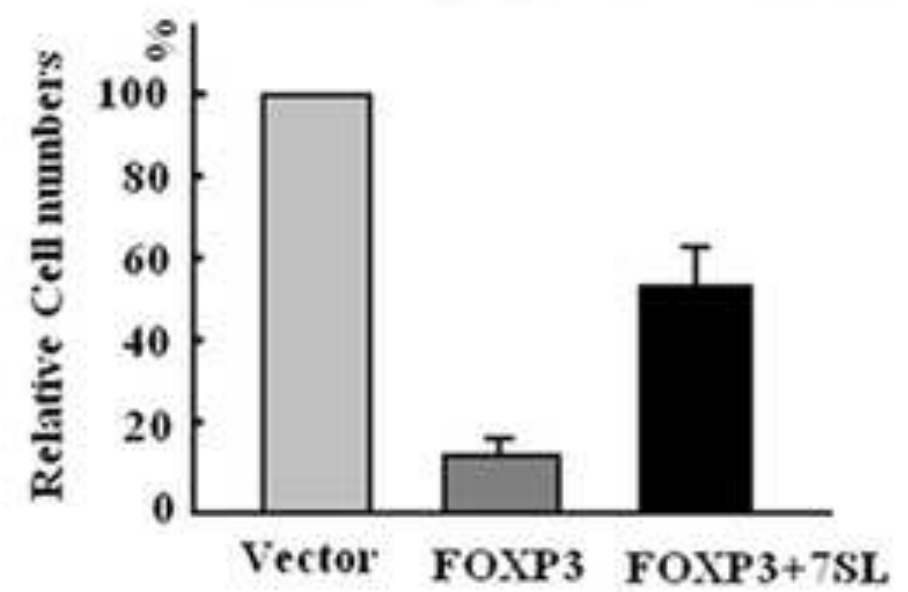

D

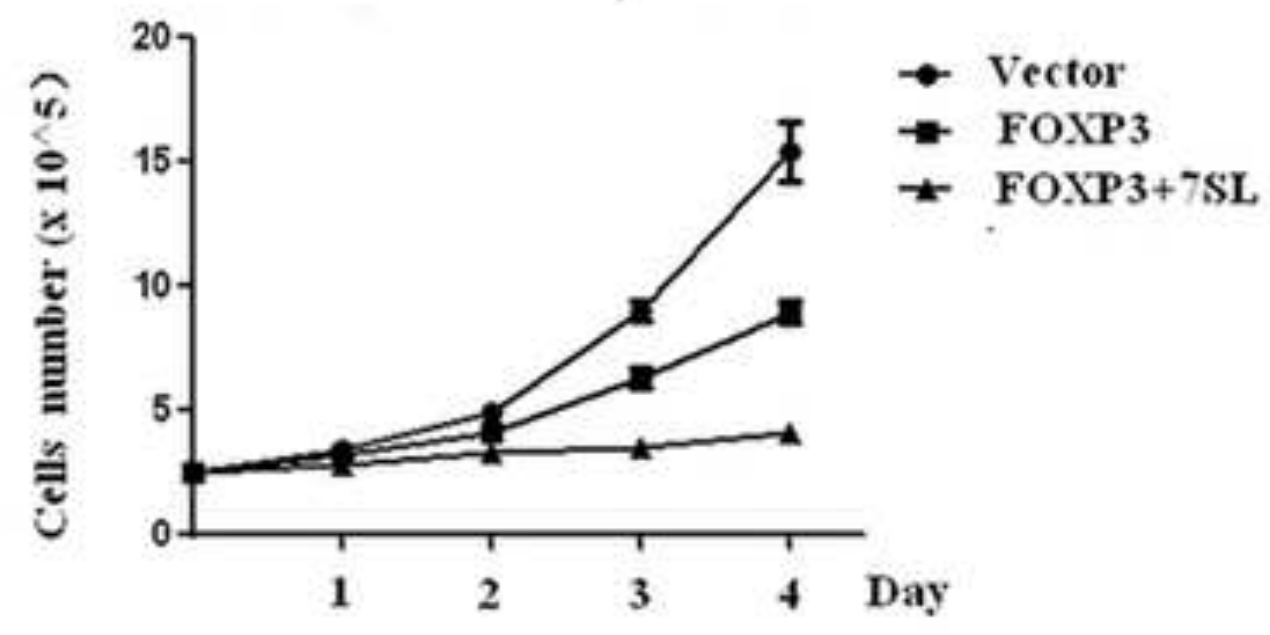


A Scramble shFoXP3-1 shFOXP3-2
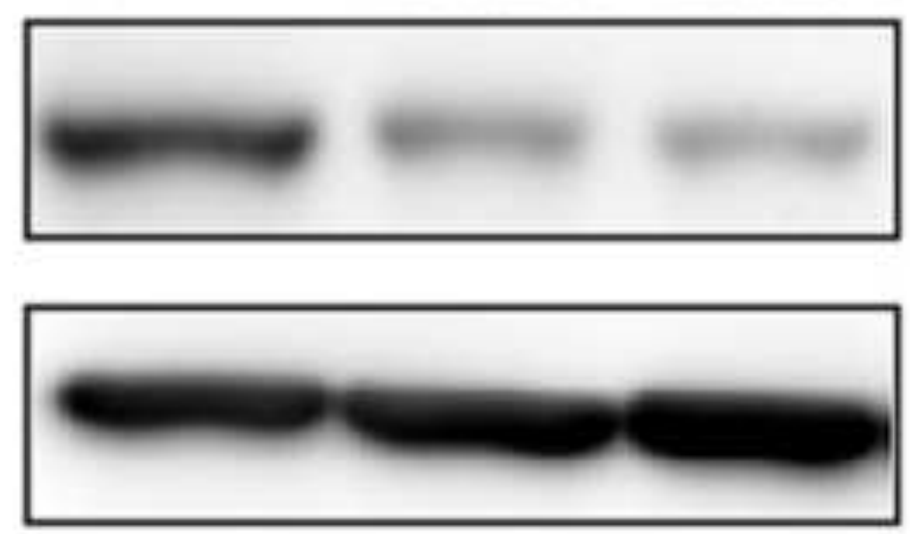

p-actin
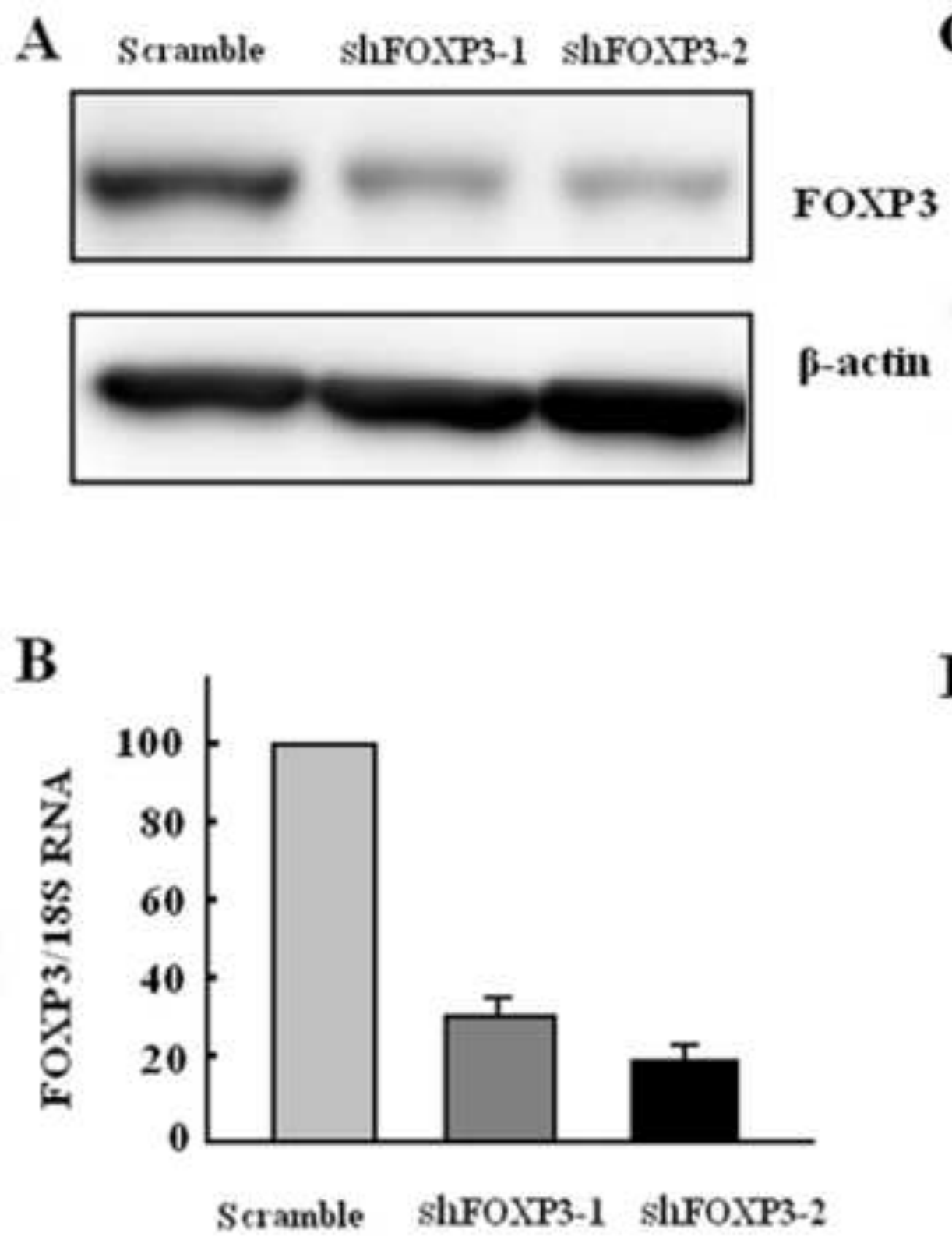

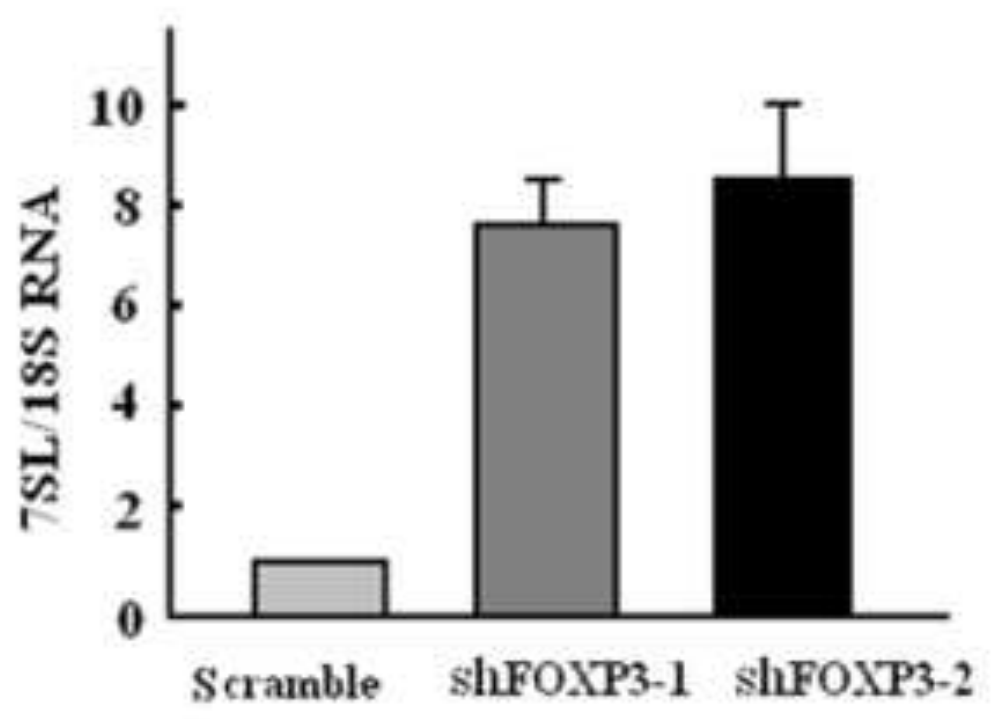

D

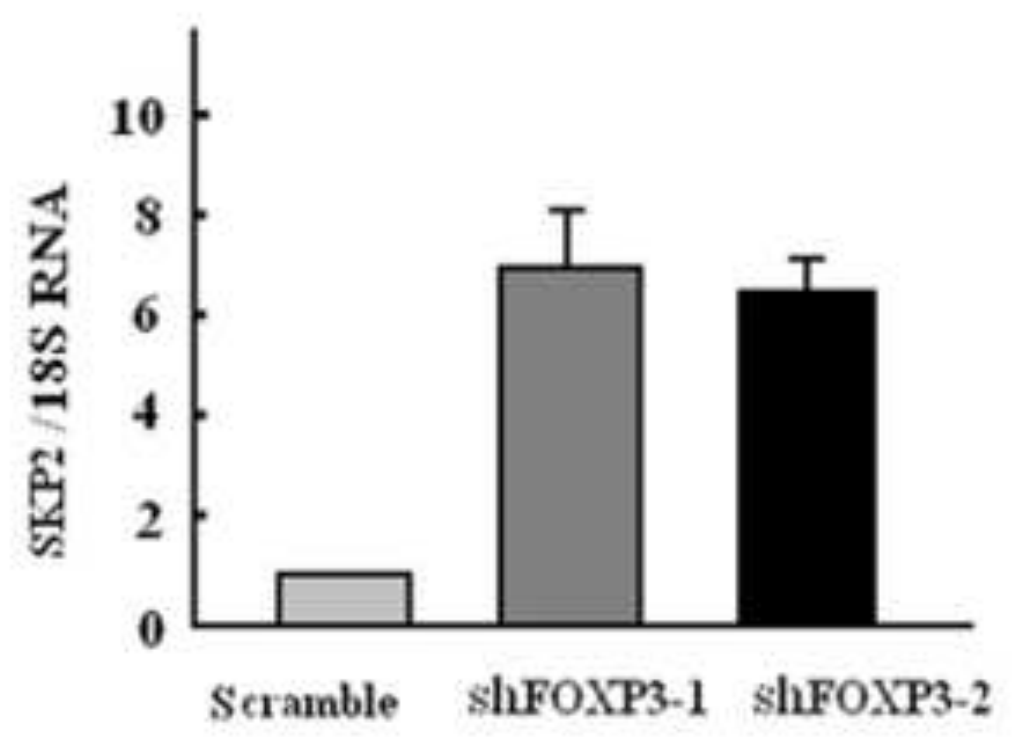


A ForkheadHNF-3 domain DNA binding Core Motif RYMLAYA and TRTKTRC

$(\mathrm{R}=\mathrm{A} / \mathrm{G}: \mathrm{M}=\mathrm{A} / \mathrm{C} ; \mathrm{Y}=\mathrm{C} / \mathrm{T}, \mathrm{K}=\mathrm{G} / \mathrm{T}$

I FOXP3 Binding Site (FBS) ChIP real time PCR prodacts

Transcription Start Site (TSS)

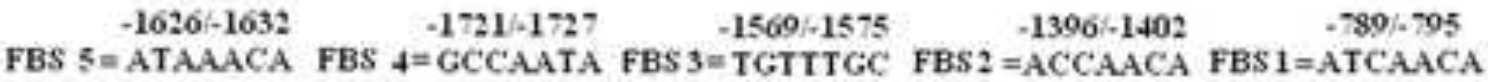

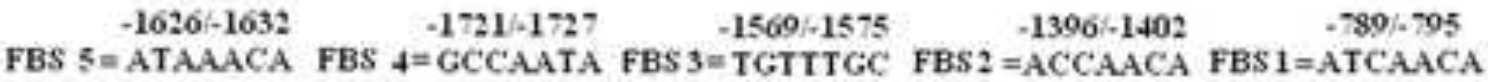

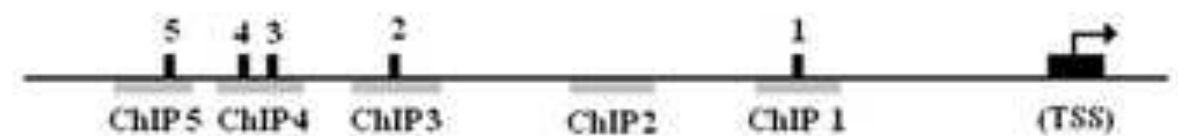

B

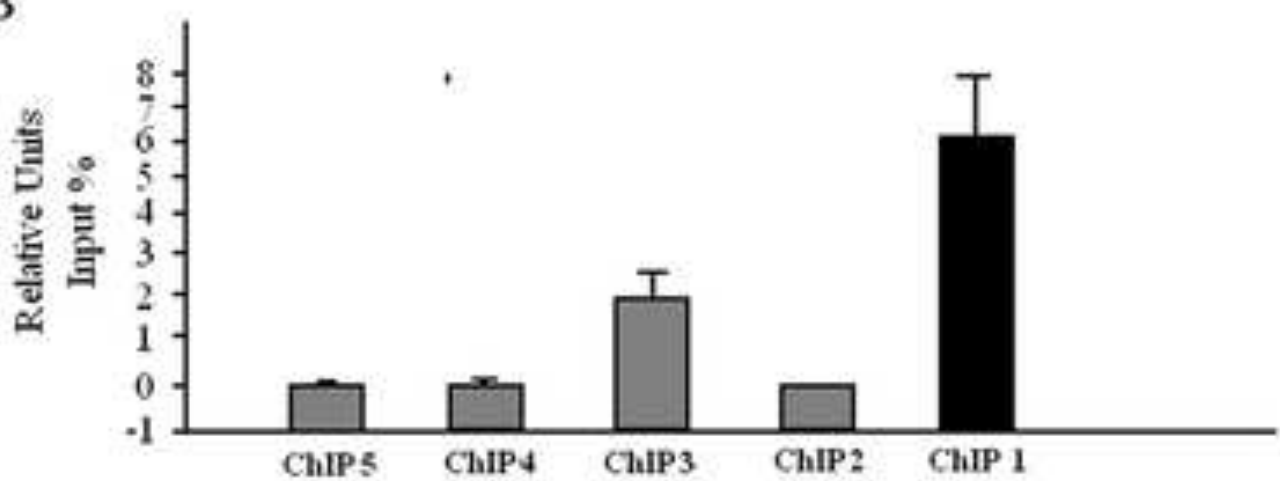

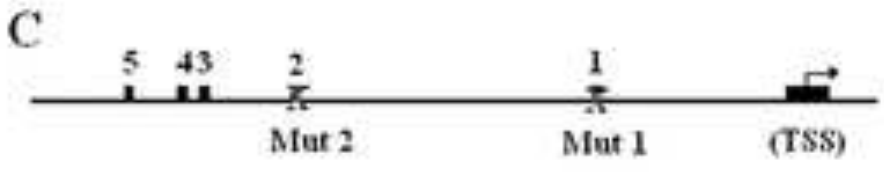

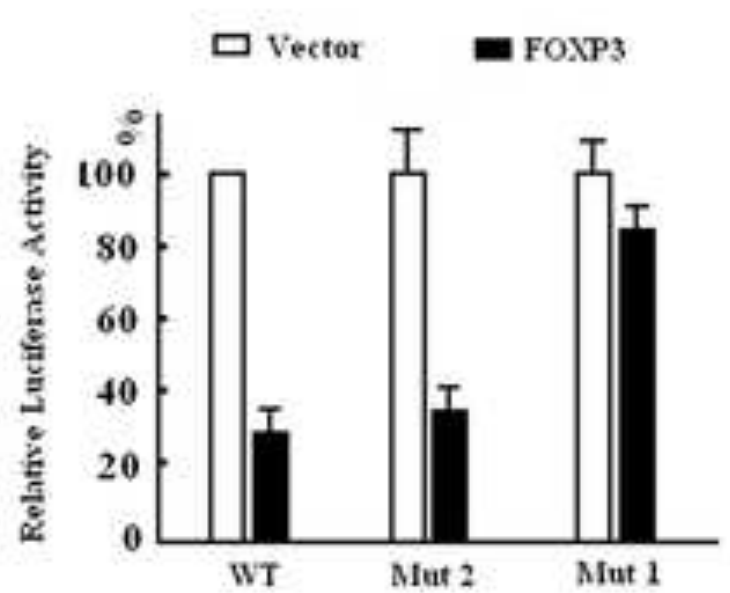


A

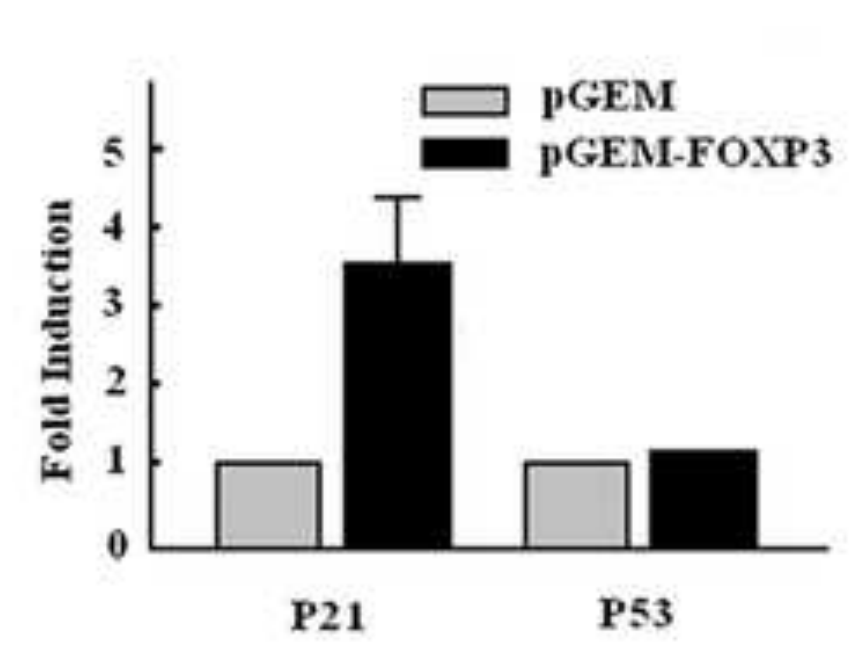

P21
B

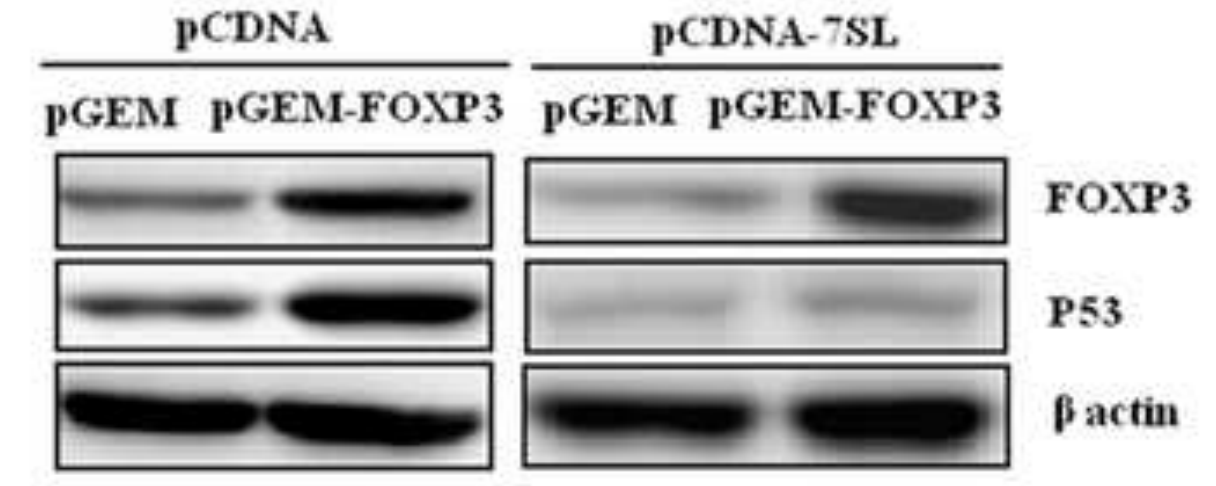

